\title{
SketchUp como ferramenta de ensino- aprendizagem na disciplina de geometria descritiva
}

\section{SketchUp as a teaching-learning tool in the discipline of descriptive geometry}

\author{
Michel Silvestre de Souza \\ Mestre em Design \\ UNESP, Bauru, Brasil \\ michel.silvestre@unesp.br \\ Roberto Alcarria dos \\ Nascimento \\ Doutor em Educação \\ UNESP, Bauru, Brasil \\ alcarria@faac.unesp.br

\section{Maria Antônia Benutti} \\ Doutora em Engenharia Civil \\ UNESP, Bauru, Brasil \\ mariabenutti@gmail.com
}

\section{RESUMO:}

Este trabalho tem como objetivo apresentar uma nova ferramenta de ensino na aprendizagem de geometria. 0 desenvolvimento da visão espacial é o grande objetivo das disciplinas de Geometria Descritiva, porém é a grande dificuldade dos alunos no ensino tradicional, assim é entendido que necessita haver uma mudança no modo de ensinar. Com o avanço tecnológico, muitos softwares de modelagem tridimensional estão sendo desenvolvidos e inseridos no ensino gráfico, o que pode ser um grande recurso no desenvolvimento do ato mental de visualizar. Muitos softwares CAD já são utilizados no ensino, e surgem constantemente softwares educacionais e softwares freeware que são mais acessíveis aos estudantes. Dentre esses programas, o SketchUp, se torna uma poderosa tecnologia para o entendimento da visualização tridimensional e um potencializador juntamente com o método tradicional de ensino, devido a sua facilidade e rapidez na modelagem, além de ser um software free e idioma compatível. A Geometria Descritiva é importante, pois desenvolve a habilidade da percepção espacial nos alunos, e a tecnologia se mostra uma ferramenta útil no ensino, sendo o SketchUp um dos programas para essa alteração no método de ensino. Procurou-se identificar as desvantagens do CAD e as vantagens do SketchUp no ensino.

Palavras-chave: geometria descritiva; ensino; sketchup; visão espacial; tecnologia.

\begin{abstract}
:
This work aims to present a new teaching tool in geometry learning. The development of spatial vision is the ultimate goal of the subjects of descriptive geometry, but it is the great difficulty of students in traditional education, so it is understood that there needs to be a change in the way of teaching. With technologic a advancement, many three-dimensional modeling software is being developed and inserted into the graphic education, which can be a great resource in developing the mental act of view. Many CAD softwares are already being used in teaching. In addition, educational and freeware softwares that are more accessible to students are constantly being created. Among these programs, SketchUp, becomes a powerful technology for understanding the threedimensional visualization and a potentiating along with the traditional method of teaching, due to its ease and speed of modeling, besides being a free and compatible language software. Descriptive geometry is important as it develops the ability of spatial perception in students, and the technology proves a useful tool in teaching, and the SketchUp one of the programs for this change in teaching method. We sought to identify the disadvantages of CAD and SketchUp advantages in teaching.
\end{abstract}

Keywords: descriptive geometry; education; sketchup; spatial vision; technology. 


\section{INTRODUÇÃO}

Este trabalho tem como objetivo apresentar uma nova ferramenta de ensino na aprendizagem de geometria. 0 desenvolvimento da visão espacial é o grande objetivo das disciplinas de Geometria Descritiva, porém é a grande dificuldade dos alunos no ensino tradicional, assim é entendido que necessita haver uma mudança no modo de ensinar.

Com o avanço tecnológico, muitos softwares de modelagem tridimensional estão sendo desenvolvidos e inseridos no ensino gráfico, o que pode ser um grande recurso no desenvolvimento do ato mental de visualizar. Muitos softwares CAD já são utilizados no ensino, e surgem constantemente softwares educacionais e softwares freeware que são mais acessíveis aos estudantes. Dentre esses programas, o SketchUp, se torna uma poderosa tecnologia para o entendimento da visualização tridimensional e um potencializador juntamente com o método tradicional de ensino, devido a sua facilidade e rapidez na modelagem, além de ser um software free e idioma compatível.

A Geometria Descritiva é importante, pois desenvolve a habilidade da percepção espacial nos alunos, e a tecnologia se mostra uma ferramenta útil no ensino, sendo o SketchUp um dos programas para essa alteração no método de ensino. Procurou-se identificar as desvantagens do CAD e as vantagens do SketchUp no ensino.

\section{DESENVOLVIMENTO}

\subsection{Geometria Descritiva e Visão Espacial}

Idealizada pelo matemático francês Gaspard Monge em meados do século XVIII, (ALVES et al, 2011, p.2) a Geometria Descritiva tem como suas principais características e objetivos, a contribuição para o desenvolvimento da visualização e do raciocínio espacial (VIANNA; TOSTE; COUTINHO, 2007, p.5). Esta disciplina está inserida dentro da grade curricular de vários cursos como engenharias, arquitetura, design, matemática.

Definiu-se como percepção visio-espacial a habilidade cognitiva de perceber e compreender formas tridimensionais e expressá-las em representações bi e tridimensionais através de meios gráficos manuais e/ou digitais. 0 sujeito com percepção visio-espacial desenvolvida é capaz de imaginar, representar e interpretar representações gráficas de formas tridimensionais em posicionamentos e visualizações diversas, como também em suas partes não visíveis (COELHO; REGO, 2007, p. 4).

Segundo Alves et al (2011), a visão espacial é uma habilidade mental de visualizar, seu aprendizado está localizado no lado direito do cérebro, que é o lado criativo, instintivo, fantasioso. Uma das capacidades do hemisfério direito é formar imagens em nossa mente e imagina-las, são aptidões importantes para o desenho (EDWARDS, 2010), sendo assim quanto mais recreativo for a aplicação do método no ensino, mais rápida é a assimilação.

No ensino superior a principal proposta é fazer com que os alunos compreendam e assimilem a visão espacial representando objetos de três dimensõe sem um plano bidimensional a partir de projeções ortogonais e vistas isométricas, como mostra a figura 1. 
Figura 1: Vistas ortogonais e isométrica
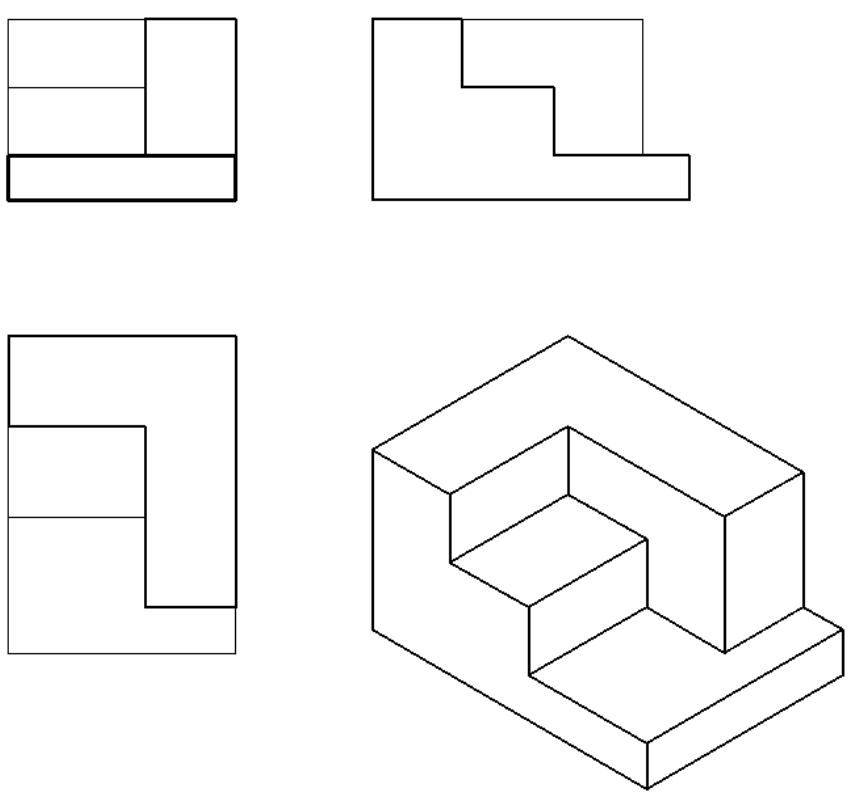

Fonte: Próprio autor (2015)

\subsection{Ensino de desenho no Brasil}

O ensino regular de desenho e geometria no Brasil começou a ocorrer no fim do século VXIII e foi se ampliando devido ao desenvolvimento industrial (BARBOSA; CHENG, 2007) e a necessidade da formação de pessoal que garantisse o funcionamento do Estado (NASCIMENTO, 1994). Nas primeiras décadas do século XX, o desenho no curso secundário não deveria atrapalhar a evolução do aluno para as séries seguintes, apenas a frequência era considerada. Assim, as aulas de desenho começaram a ser menosprezadas, já que nunca reprovavam (NASCIMENTO,1994). Em 1931, o curso secundário foi dividido em fundamental e complementar, sendo o fundamental uma formação básica geral e o complementar uma preparação para o ingresso no curso superior, que posteriormente foi alterado de nome para curso ginasial, mas sempre o desenho fez parte de alguma forma e com vários momentos e valores educativos (NASCIMENTO, 1994).

Na década de 70, depois de uma mudança na diretriz de ensino, o desenho deixou de fazer parte dos níveis fundamental e médio, sendo estudado apenas em cursos técnicos e algumas escolas mais tradicionais, gerando futuros problemas em alunos ingressantes na graduação. Os discentes em cursos como engenharia e arquitetura acabam por ter muita dificuldade na compreensão devido à falta de embasamento (BARBOSA; CHENG, 2007) e deficiência na visualização do objeto tridimensional no espaço (MACHADO, 2011). Em sua pesquisa, Machado (2011) mostra que a maioria dos alunos de engenharia nunca havia estudado desenho no ensino fundamental ou médio como é representado na figura 2. 
Figura 2: Resultado da pesquisa sobre estudo previo de desenho

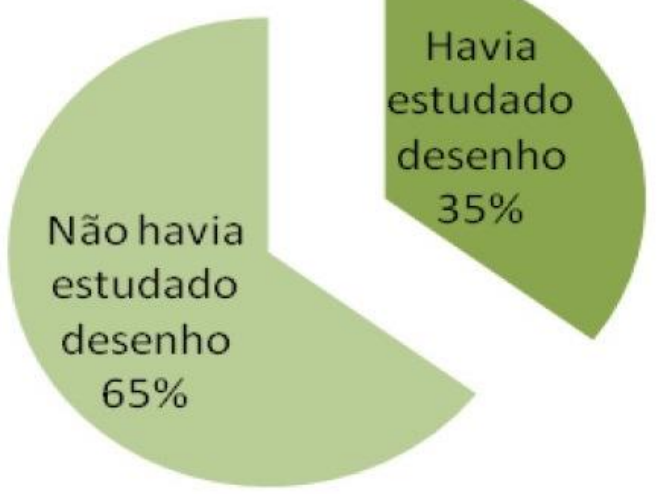

Fonte: MACHADO (2011)

Atualmente, o ensino de Geometria Descritiva no ensino superior, se dá na maioria das vezes no modo tradicional ou convencional, que são aulas expositivas e práticas, porém demonstra ser um método ineficiente (LIMA; CARVALHO; ASEVEDO, 2007) pois se percebe que os alunos demonstram ter muita dificuldade no aprendizado. o professor explica o conteúdo em lousa e/ou data show, e os alunos representam o objeto em folhas no formato A3 com seus instrumentos como prancheta, lapiseiras, esquadros, régua T, compassos, etc. No aprendizado, a primeira etapa é apresentado um objeto tridimensional e o discente necessita representar o objeto em projeções ortogonais (vistas frontal, superior e lateral esquerda), uma segunda etapa, o aluno representa em perspectiva isométrica um objeto a partir das vistas ortogonais. Esse processo requer treinamento e desenvolve a capacidade de visualização dos alunos (ARAÚJO, 2014). No método convencional, uma maneira eficiente de explicar a tridimensionalidade é utilizando blocos de sabão ou espumas de floricultura, figura 3, onde a segunda é mais prática a modelagem e mais fácil de esculpir. Os blocos esculpidos didaticamente servem como apoio para realizar as primeiras vistas ortogonais, facilitando a visualização das peças.

Figura 3: Espuma floral

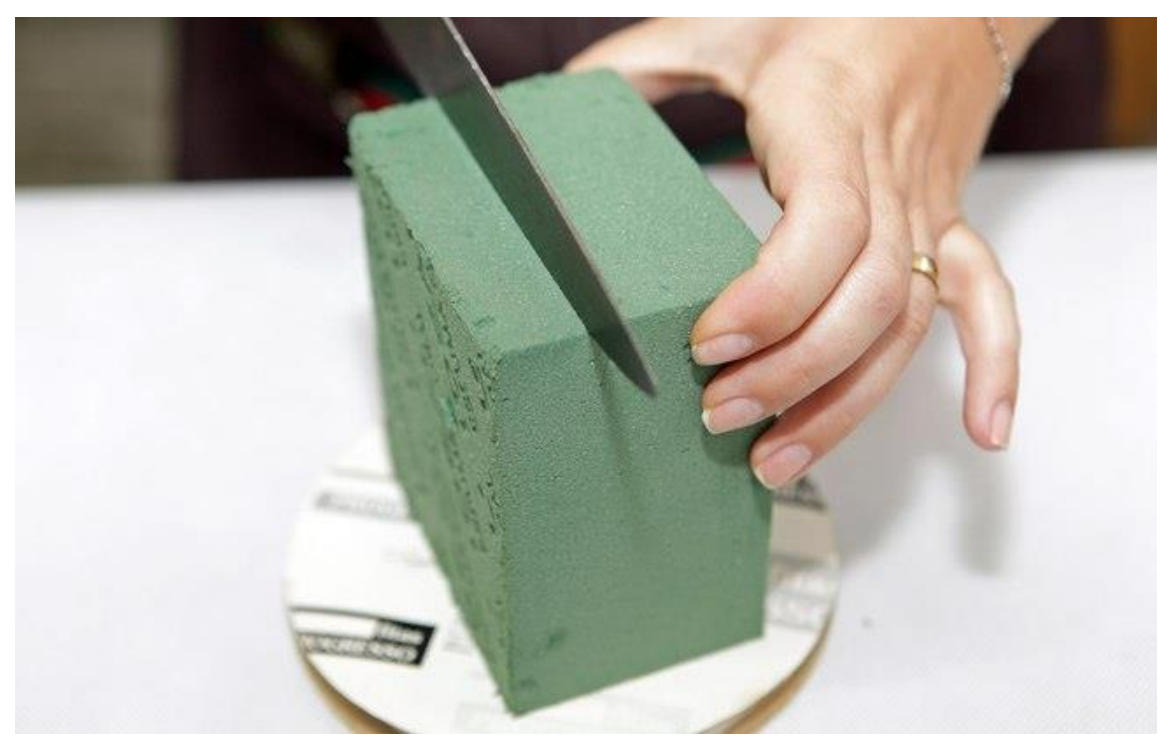

Fonte: Próprio autor (2015) 
Nas universidades, muitos cursos como engenharia, arquitetura e design, sofreram grande desvalorização no ensino de expressão gráfica, devido à redução de carga horária em várias disciplinas (BARBOSA; CHENG, 2007), em especial de desenho, inserção do uso da tecnologia no ensino da geometria, falta de espaço físico para salas de desenho e criação de laboratórios de informática, elevado número de alunos em sala de aula, principalmente em instituições particulares (MACHADO, 2011), e o novo perfil dos discentes, com uma geração que já crescem utilizando ferramentas digitais aliados a internet (LIMA; CARVALHO; ASEVEDO, 2017). Na maioria das vezes os alunos chegam nas salas de aula já questionando a utilidade da disciplina, visto que já tem conhecimento da existência de softwares gráficos que realizam o mesmo trabalho.

As novas gerações dominam melhor o computador (graças aos sitesna internet, kits multimídia, aprendizado à distância de programasgráficos via web, etc.) e muitas vezes resistem um pouco mais em desenhar à mão livre ou com instrumentos convencionais de desenho (MACHADO, 2011, p. 10).

As principais dificuldades no ensino de geometria foram listadas resumidamente para melhor entendimento, conforme bibliografia consultada:

- Alunos sem os conhecimentos mínimos necessários em geometria;

- Desvalorização da disciplina;

- Redução na carga horária das disciplinas;

- União dos métodos tradicionais e digitais em uma só disciplina;

- Falta de espaço físico para salas de desenho com pranchetas e salas de informática;

- Falta de motivação dos alunos, pois novas gerações já estão integradas com as tecnologias digitais.

- Deficiência na visualização espacial e tridimensional de objetos.

\section{TECNOLOGIA COMO FERRAMENTA DE ENSINO}

0 computador e as mídias digitais são recursos ainda pouco explorados como ferramenta no ensino da geometria (LIMA; CARVALHO; ASEVEDO, 2007). Com o avanço tecnológico muitos softwares foram criados, principalmente na área de computação gráfica, que auxiliam no desenvolvimento da visão espacial, tornando a disciplina menos exaustiva, menos desestimulante e incentivando que seja atrativa e agradável. Nota-se que a informática tem se tornado mais acessível (ALVES et al, 2011), e tem se formado uma nova geração de jovens crescendo utilizando esses recursos e alterando o perfil do aluno, que tem preferido o uso dessas mídias aos métodos tradicionais.

Machado (2011), afirma que em questionários aplicados aos alunos $60 \%$ prefere o método digital no ensino, como é apresentado o gráfico na figura 4. 
Figura 4: Resultado da pesquisa sobre preferência de métodos no ensino de desenho

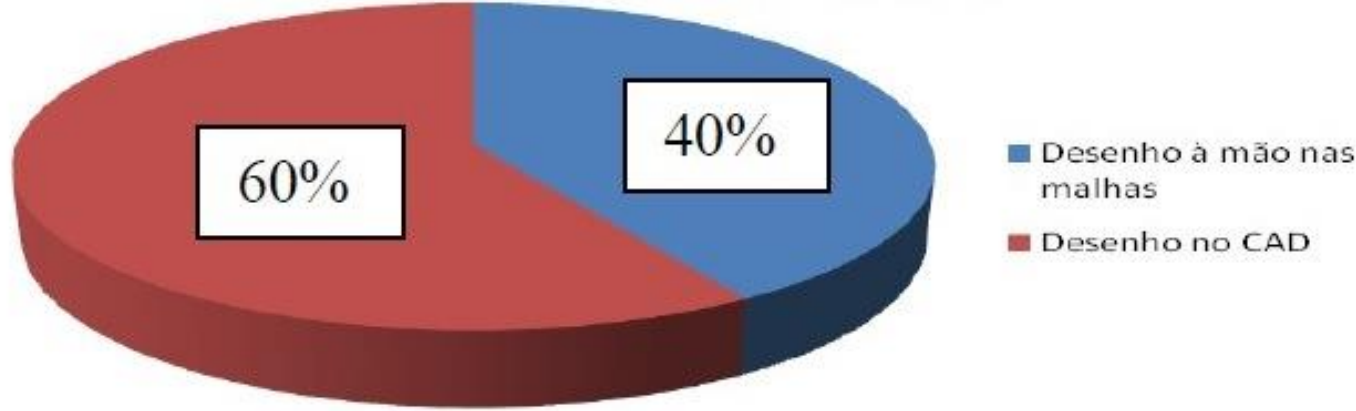

Fonte: MACHADO (2011)

A inserção da computação gráfica, principalmente a modelagem 3D, assume papel importante no ensino do espaço e da forma, potencializando juntamente com o método tradicional os recursos necessários para a assimilação da visão espacial, além de serem altamente difundidos e acessíveis aos ingressantes das universidades (ARAÚJO, 2014).

\subsection{Softwares CAD}

O CAD, desenho assistido por computador, são programas de computação gráfica que permitem criar e editar desenhos em ambientes bi e tridimensionais. Muitas áreas utilizam esses programas, engenharias civil, mecânica e elétrica, arquitetura, design.

O software mais conhecido e utilizado é o AutoCad, da Autodesk, empresa que domina o mercado da área gráfica há décadas sendo líder em vendas com sua suíte de programas como Inventor, Revit e 3D Studio Max. São softwares pagos, e atualmente libera licença de até três anos para estudantes, porém a dificuldade em fazer download e instalação acaba fazendo com que os discentes optem pela versão pirateada.

0 ensino de CAD nos cursos de engenharia começou no final da década de 80 , mas a tecnologia só foi aceita de fato no final da década de 90 .

Devido às vantagens do uso do $\mathrm{CAD}$ como rapidez e precisão, a tecnologia difundiu-se no mercado de trabalho, evoluiu com o passar dos anos de forma acelerada, causando uma série de mudanças na prática profissional. Já inserido no ensino de geometria, os professores passam por outros problemas como as dificuldades do software (MORAIS, 2010).

Coelho e Rego (2007) relataram em suas pesquisas as dificuldades do uso do CAD no ensino da geometria, e indica que as futuras pesquisas serão apenas com o uso do SketchUp.

As principais desvantagens dos CADs no ensino de geometria:

- Software com licença paga;

- Difícil acesso dos alunos aos softwares;

- Idioma inglês;

- Ferramentas que necessitam de um tempo de curso;

- Precisa de mais tempo de trabalho para assimilar o software. 


\subsection{Softwares freeware e educacionais}

Softwares freeware são aqueles que possuem licença livre para seu uso, trazendomuitas vantagens, principalmente em seu custo (BARBOSA; CHENG, 2007). Existem vários aplicaticos educacionais freeware para o ensino de geometria, Alves et al (2011) e Lima, Carvalho e Asevedo (2007), destacaram alguns como o GD, Hypergeo, AEIOU, Teoria Geral das Projeções, Jogo de Paciência de Planos, Espaço GD e Visual GD. Muitas universidades apresentam problemas na falta de investimentos, tanto em equipamentos, quanto em softwares, assim o uso de CAD freeware reduz muito nos custos de implantação, além de facilitar a utilização por parte dos alunos em seus computadores pessoais, não necessitando ter softwares pirateados, reduzindo a ilegalidade (BARBOSA; CHENG, 2007).

Em relação ao CAD, Barbosa e Cheng (2007) levantaram uma lista de softwares free nos modos 2D e 3D, que podem ser utilizados tanto por estudantes como por profissionais.

Tabela 1: Relação de CAD freeware

\begin{tabular}{|c|c|c|c|}
\hline \multicolumn{4}{|l|}{ 2D } \\
\hline Nome & Empresa & Licença & Site \\
\hline A9CAD & A9 Tech & Freeware & http://www.a9tech.com \\
\hline CadStd & John Apperson & Freeware & http://www.apperson.org/cadstd/ \\
\hline Delta CAD & Cypress Software & Trial & http://www.deltacad.com \\
\hline DESI-III & H. Mariën & Freeware & http://users.pandora.be/desi-iii/index.html \\
\hline FelixCAD 5 LT & GiveMePower & Freeware & http $/ /$ www.givemepower.de \\
\hline Free 2Desing & Think3 & Freeware & http://www.free2design.org \\
\hline JustCad & Jon Hoke & Freeware & http $/ /$ www.justcad.com \\
\hline QCad & RibbonSoft GmbH & $\begin{array}{l}\text { Código } \\
\text { Aberto }\end{array}$ & http://www. qcad.org \\
\hline Solid Edge Free & UGS & Freeware & www.solidedge.com/free2d/ \\
\hline \multicolumn{4}{|l|}{ 3D } \\
\hline Nome & Empresa & Licença & Site \\
\hline 3D Canvas & Amabilis & Freeware & www. amabilis.com \\
\hline Blender & Blender Foundation & $\begin{array}{l}\text { Código } \\
\text { aberto }\end{array}$ & http://www.blender.org \\
\hline BRL-CAD & Governo americano & $\begin{array}{l}\text { Código } \\
\text { Aberto }\end{array}$ & http://sourceforge.net/projects/brlcad/ \\
\hline CADX11 & GrayTech & Freeware & 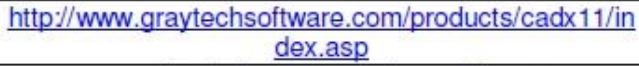 \\
\hline DesignCAD & IMSI & Trial & www.imsisoft.com/faminfo.asp?fam=2 \\
\hline DesignWorkshop & Artifice & Freeware & http://www.artifice.com/ \\
\hline Free CAD & Aik-Siong Koh & Freeware & www. askoh.com \\
\hline Google SketchUp & & Freeware & http://sketchup.google.com/download.html \\
\hline Minos CAD 2.2 & John Apperson & Freeware & www.le-boite.com/downl.htm \\
\hline My3DEngine & i-compute & Freeware & www.i-compute.net/projects My3DEngine.php \\
\hline Turbo CAD & IMSI & Trial & www.turbocad.com \\
\hline VectorEngineer & & Freeware & http $/ /$ www.vectorengineer.com/ \\
\hline WorkCAD & CIENGE & Trial & $\frac{\text { http://www.workcad.com.br/index.php?session }=\text { dow }}{\text { nload trial }}$ \\
\hline
\end{tabular}

Fonte: BARBOSA; CHENG (2007)

\subsection{SketchUp}

SketchUp é um software de propriedade da Trimble, desenvolvido para a criação de modelos 3D, possuindo versões disponibilizadas nas formas profissional e gratuita, sendo amplamente difundido e utilizado por arquitetos e engenheiros na execução de maquetes eletrônicas (VIANNA; TOSTE; COUTINHO, 2007). Mesmo não possuindo função didática, pode ser muito aproveitado para o desenvolvimento da 
percepção espacial dos alunos devido sua facilidade no manuseio das ferramentas com poucas orientações (ARAÚJO, 2014).

0 programa, fácil encontrar na internet (http://www.sketchup.com/ptBR/download) e de simples instalação, é versátil e modela os objetos diretamente no ambiente 3D, suas ferramentas são intuitivas e diretas, fazendo a modelagem rápida e instantânea. Com o modelo pronto é possível mudar a visualização de maneira fácil. 0 movimento de transição entre as vistas é dinâmico e permite a assimilação por parte do aluno, assim como acontece didaticamente nos blocos de sabão e espuma floral,mas nesse caso acontece virtualmente, o modelo se move até que a vista desejada seja exibida. Esta facilidade atua na percepção de representação dos alunos (ARAÚJO, 2014). Em relação as normas de representação do desenho técnico, as linhas já são configuradas de acordo com a profundidade, as mais próximas do ponto de visão têm espessuras grossas e as mais distantes são mais finas.

Figura 5: Interface SketchUp 2014

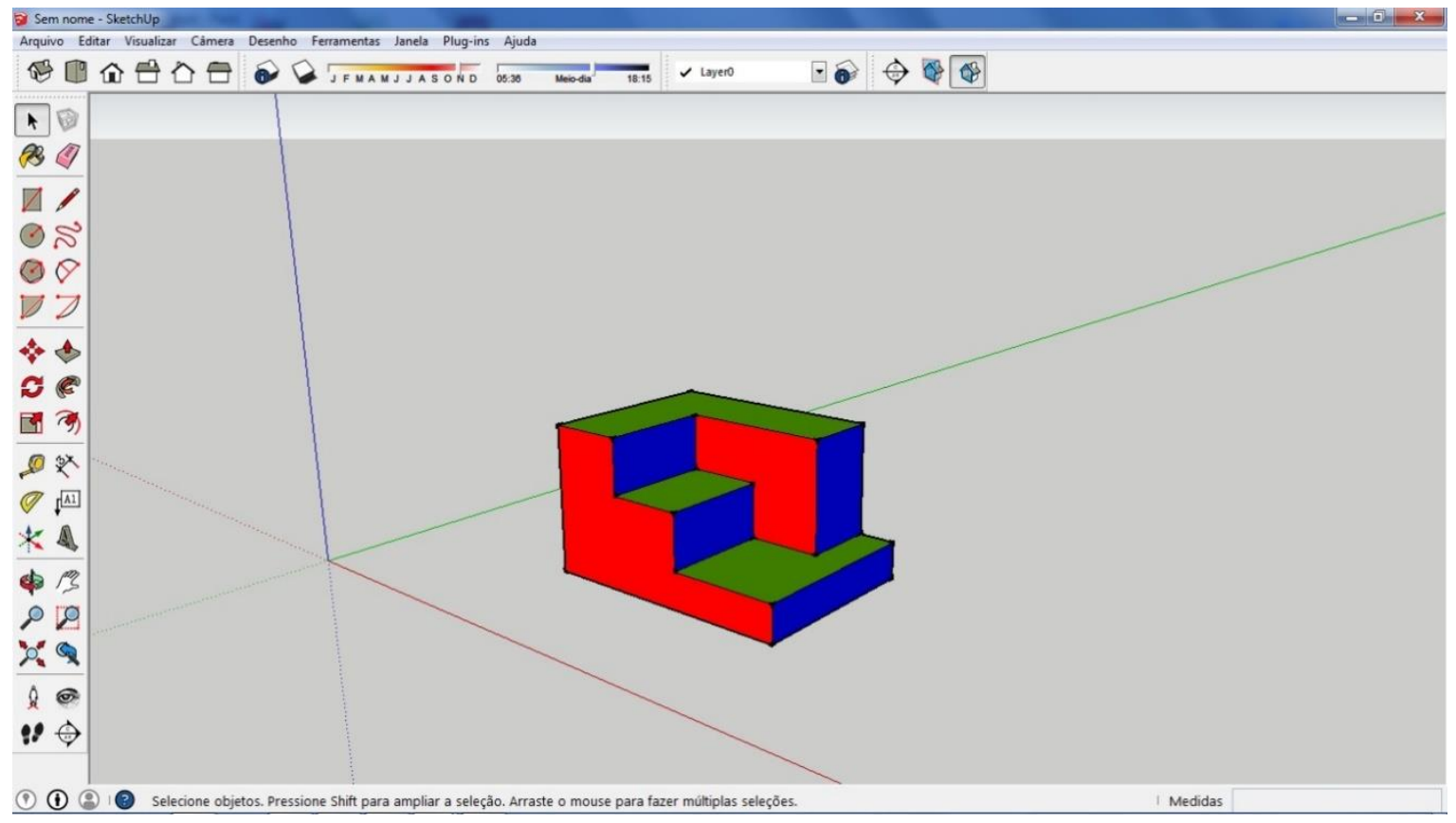

Fonte: Próprio autor (2015)

De acordo com as referências bibliográficas destacam-se as principais vantagensdo SketchUp no ensino da Geometria Descritiva:

- Software gratuito;

- Interface e ferramentas intuitivas;

- Idioma compatível;

- Atua diretamente no ambiente 3D;

- Movimentação Instantânea dos modelos;

- Versátil e dinâmico;

- Rápido e fácil de modelar;

- Não necessita de curso específico;

- Fácil acesso e instalação do software;

- Com pouco tempo de uso já se aprende a manusear as ferramentas. 


\section{CONCLUSÃO}

Procurou-se identificar as principais dificuldades dos alunos no aprendizado em Geometria Descritiva no ensino tradicional e indicar uma nova ferramenta para o auxílio da assimilação da visualização tridimensional. A visão espacial pode ser melhorada por meio de uso de softwares, facilitando o entendimento. Aproveitando as tecnologias digitais, o SketchUp foi identificado como possível ferramenta freeware, que trabalha diretamente no ambiente 3D. É grande a facilidade na modelagem dos objetos, intuitivo, rápido e dinâmico.

É notável que é tempo de mudanças no ensino de geometria, a informática vem sendo um ótimo recurso na melhoria do aprendizado em todas as áreas. Com a computação gráfica em alta, é possível encontrar outras formas de transmitir osconceitos utilizando softwares free e com modelagem tridimensional. O SketchUp pode ser estudado mais a fundo em outras pesquisas, sendo aplicado em sala de aula e relatando estas experiências.

\section{REFERÊNCIAS BIBLIOGRÁFICAS}

ALVES, Maria da Conceição Amaral; COSTA, Ivoneide de França; CARDOSO, Christina Araújo Paim. A Geometria Descritiva e a Tecnologia Computacional. In: Simpósio Nacional de Geometria Descritiva e Desenho Técnico. 20, e International Conference on Graphics Engineering for Arts and Design. 9, 2011, Rio de Janeiro. Anais... Rio de Janeiro, 2011.

ARAÚJ0, Thiago Thielmann de. Utilização de Softwares de Modelagem Tridimensional como Ferramenta Didática de Desenho Técnico. In: CongressoBrasileiro de Educação em Engenharia. 42, 2014, Juiz de Fora. Anais... Minas Gerais,2014.

BARBOSA, Carolina Chaves; CHENG, Liang-Yee. Uso de CAD Freeware no Ensinode Engenharia. In: Simpósio Nacional de Geometria Descritiva e Desenho Técnico. 18,e InternationalConferenceonGraphicsEngineering for Artsand Design. 7, 2007,Curitiba. Anais... Paraná, 2007.

COELHO, Elizete da Silva; REGO, Rejane de Moraes. Percepção VisioEspacial:Combinando Representação Gráfica e Modelagem 3D. In: Simpósio Nacional de Geometria Descritiva e Desenho Técnico. 18, e International Conference on Graphics Engineering for Arts and Design. 7, 2007, Curitiba. Anais... Paraná,2007.

EDWARDS, Betty. Desenhando com o lado direito do cérebro. 2 ed. Rio de Janeiro: Ediouro, 2010.

LIMA, Mônica Maria Fernandes de; CARVALHO, Sheila Oliveira de; ASEVEDO, Laíze Fernandes de. Informatizando e Modelando a Geometria Gráfica. In: Simpósio Nacional de Geometria Descritiva e Desenho Técnico. 18, e International Conference on Graphics Engineering for Arts and Design. 7, 2007, Curitiba. Anais... Paraná, 2007.

MACHADO, Silvana Rocha Brandão. A Compatibilização do Ensino Tradicional de Desenho com as Novas Tecnologias. In: Simpósio Nacional de Geometria Descritiva eDesenho Técnico. 20, e International Conference on Graphics Engineering for Arts and Design. 9, 2011, Rio de Janeiro. Anais... Rio de Janeiro, 2011. 
MORAIS, Luciana Klein da Silva de. AutoCAD 2010 2D básico. Santa Cruz do Rio Pardo: Editora Viena, 2010.

NASCIMENTO, Roberto Alcarria do. 0 ensino do desenho na Educação brasileira: apogeu e decadência de uma disciplina escolar. Marília: 1994. 120p. Dissertação (mestrado em Educação). Faculdade de Filosofia e Ciências. UNESP.

VIANNA, Sonia Sá; TOSTE, Berta López; COUTINHO, Rodrigo Ribeiro. O Desenho eas Novas Tecnologias no Colégio Pedro II: Um Enfoque Atual. In: Simpósio Nacionalde Geometria Descritiva e Desenho Técnico. 18, e International Conference on Graphics Engineering for Arts and Design. 7, 2007, Curitiba. Anais... Paraná, 2007. 